\title{
Tone Discrimination as a Window Into Acoustic Perceptual Deficits in Parkinson's Disease
}

\author{
Joshua Troche ${ }^{\mathrm{a}}$, Michelle S. Troche ${ }^{\mathrm{a}}$, Rebecca Berkowitz ${ }^{\mathrm{b}}$, Murray Grossman $^{\mathrm{c}}$, and Jamie \\ Reilly ${ }^{\mathrm{a}}$ \\ aUniversity of Florida, Gainesville \\ bTemple University, Philadelphia, PA \\ 'University of Pennsylvania School of Medicine, Philadelphia
}

\begin{abstract}
Purpose-Deficits in auditory perception compromise a range of linguistic processes in persons with Parkinson's disease (PD), including speech perception and sensitivity to affective and linguistic prosody. An unanswered question is whether this deficit exists not only at the level of speech perception, but also at a more pervasive level of auditory perception. It is possible that PD produces a selective impairment in the perception of a salient acoustic feature such as frequency, amplitude, or duration.
\end{abstract}

Method-Auditory perception in persons with PD was investigated using a tone discrimination task where clients $(N=12)$ and age-matched controls $(N=15)$ made same/different judgments for pairs of pure tones that were factorially varied by acoustic feature (i.e., frequency, amplitude, or duration) crossed with perceptual distance (i.e., close vs. far).

Results-Relative to healthy age-matched controls, persons with PD showed marked impairment in tone discrimination. Persons with PD showed an acoustic feature by perceptual distance interaction that was characterized by deficits in detecting frequency and amplitude differences for perceptually near tones.

Conclusion-These results suggest that persons with PD show a reduced ability to notice change in frequency and amplitude as compared to normal older adults. More generally, these findings implicate a frontal-striatal contribution to auditory perception.

\section{Keywords}

Parkinson's disease; speech perception; auditory perception; tone detection; frontal lobe
Parkinson's disease (PD) has been associated with a wide variety of deficits in auditory perception, including impairments in word recognition (Graber, Hertrich, Daum, Spieker, \& Ackermann, 2002), insensitivity to emotional prosody conveyed by tone of voice (Pell, 1996; Pell, Cheang, \& Leonard, 2006; Pell \& Leonard, 2003), and inability to detect syntactically relevant prosodic markers such as segmental stress patterns that mark nouns and verbs (Kotz, Schwartze, \& Schmidt-Kassow, 2009). The mechanism(s) underlying these perceptual deficits remains controversial. One unanswered question is whether this wide range of impairments reflects a focal deficit within the auditory system proper. It is conceivable, for example, that the neuropathology of PD selectively degrades a person's

\footnotetext{
(C) American Speech-Language-Hearing Association
}

Correspondence to Joshua Troche: jetgator@ufl.edu. 
perception of one or more focal acoustic properties, such as sensitivity to pitch variability or spectral complexity (for a discussion of non-motor basal ganglia functions, see Kotz et al., 2009). The insensitivity to change is a likely outcome due to the findings in previous research of persons with PD not only displaying depressed auditory, visual, and somatosensory evoked potentials (Abbruzzese \& Berardelli, 2003; Gawel, Das, Vincent, \& Rose, 1981; Muthane, Satishchandra, \& Subhash, 1993), but also demonstrating deficits in face recognition and estimation of elapsed time (Smith, Harper, Gittings, \& Abernethy, 2007; Sprengelmeyer et al., 2003). Our aim in this study was to determine if persons with PD were insensitive to subtle differences in frequency, amplitude, and duration of pure tones.

\section{A Basis for Auditory Deficits in Persons With PD}

Historically, neurologically constrained models of language perception have emphasized critical roles of the left superior temporal lobe (i.e., primary and secondary auditory cortex) in speech perception. The pioneering work of Carl Wernicke (1874), for example, stressed an essential role of superior and posterior temporal structures in processing sound (i.e., auditory images) and extracting meaning from spoken words. This paradigmatic view of temporal lobe dominance for speech perception has pervaded much of both contemporary aphasiology and the broader field of auditory cognitive neuroscience (Caspari, 2005; Damasio, 2001; Kertesz, Lau, \& Polk, 1993).

From such a temporal lobe-centric perspective, PD presents a somewhat paradoxical lesion model in that its primary pathology impacts frontal subcortical and midbrain structures (e.g., thalamus, basal ganglia; Braak, Ghebremedhin, Rub, Bratzke, \& Del Tredici, 2004). For this reason, one particular theory with relevance for audition in PD is the dual-stream model, which holds that speech perception, much like the cortical processing of vision, is subserved by two dissociable pathways: a ventral stream and a dorsal stream (Hickok \& Poeppel, 2007). The putative ventral stream is composed of a variety of superior and middle temporal lobe structures whose function includes the hierarchical decomposition of spectral and temporal elements of the speech signal (Binder et al., 2000; Binder, Frost, Hammeke, Rao, \& Cox, 1996). The dorsal stream, a lesser known pathway, is believed to encompass regions of the left inferior frontal cortex, including Broca's area and the adjacent posterior tissue (i.e., premotor cortex; Hickok, 2009). Hickok and Poeppel (2007) suggested that a primary role of the dorsal speech perception pathway is sensorimotor integration, related specifically to integrating auditory speech input with speech motor output processes. Thus, the dorsal pathway is specialized for processing input by online simulation of our own articulatory motor plans of the words that we hear (also see the motor theory of speech perception; Liberman, Cooper, Shankweiler, \& Studdert-Kennedy, 1967; McGurk \& MacDonald, 1976).

The dual-stream theory of speech perception can provide an overarching framework for generating a series of predictions about PD, whose pathology typically spares temporal lobe structures implicated in the ventral stream while compromising communication between inferior frontal lobe structures that support the dorsal stream (Demirci, Grill, McShane, \& Hallett, 1997; Klockgether, Borutta, Rapp, Spieker, \& Dichgans, 1995; Schneider, Diamond, $\&$ Markham, 1986). Thus, based on the relatively focal distribution of the frontal-striatal pathology of PD, a dual-stream model might predict declines in coding and perceptual simulation of speech sounds. However, an open question is whether such deficits have a basis that extends beyond speech-specific processing to impact more fundamental acoustic properties of sound.

Burton and Small (2006) addressed this question in a study involving a tone segmentation task. The task involved making same/different judgments regarding pure tones. It was 
determined that this tone segmentation task showed similar activation in the left middle frontal gyrus near the border of the inferior frontal gyrus as a task of speech segmentation. These results support the claim hypothesis that the frontal-striatal pathology of PD could also cause disruption in the ability to discriminate tones. Despite the implications for understanding speech perception deficits in persons with PD, few studies have investigated tone discrimination deficits in persons with PD. We do so here using a pure-tone discrimination task.

Pure tones are useful in psychoacoustic research in that they represent simple sinusoidal waves characterized by three primary parameters (i.e., frequency, amplitude, and duration). Using pure tones, it is possible to selectively manipulate these acoustic parameters (e.g., holding frequency constant while manipulating duration) with the goal of isolating a particular parameter. In order to determine if persons with PD show decreased sensitivity to change, we manipulated pairs of pure tones to be either perceptually close or far. We also made comparisons between persons with PD and age-matched controls due to an extensive literature describing increased insensitivity to change in these acoustic properties as a normal occurrence in healthy aging persons (Harris, Mills, \& Dubno, 2007; Harris, Mills, He, \& Dubno, 2008; Pichora-Fuller \& Souza, 2003).

\section{Method}

\section{Participants}

Participants included 12 adults with mild to moderate PD as defined by a Hoehn and Yahr (1967) Stage II-III classification and 15 healthy age-matched controls. The adults with PD included 7 men and 5 women ages $51-80\left(M_{\text {age }}=68.3, S D=8.7\right)$. The control group included 8 men and 7 women ages $61-80\left(M_{\mathrm{age}}=70.3, S D=5.9\right)$. Participants' global cognition and naming were within normal limits as assessed by the Mini-Mental State Examination $(M=28.58, S D=1.16$; Folstein, Folstein, \& McHugh, 1975) and the Boston Naming Test (Kaplan, Goodglass, \& Weintraub, 1983). At the time of testing, all participants were free of major depression as assessed by the Beck Depression Inventory ( $M$ $=6, S D=4.77$; Beck, Steer, \& Carbin, 1988) and had no history of other neurological disease or neurological surgery including deep brain stimulation, palidotomy, or thalamotomy. All participants were screened for hearing loss using a standard pure-tone detection procedure automated for PC presentation (Reilly, Troiani, Grossman, \& Wingfield, 2007).

\section{Materials and Procedure}

E-Prime 2.0 Professional (Psychology Software Tools) software was used to present stimuli and capture the accuracy and latency of participants' responses ( \pm 10 -ms error). Participants made same/different judgments as quickly and accurately as possible for pairs of pure tones that were presented aurally at a comfortable volume for three separate experimental blocks of roughly equal duration. These experimental blocks were counterbalanced, and stimuli within blocks were completely randomized. At the level of an individual trial (each consisting of a pair of pure tones), the interstimulus interval (ISI) was $750 \mathrm{~ms}$ and the intertrial interval was $1500 \mathrm{~ms}$.

Each experimental block involved selective manipulation of one acoustic parameter (i.e., frequency, amplitude, or duration) while holding the other two parameters constant. Within each block, participants heard 32 pairs of pure tones in completely random order. Half ( $n=$ 16) of the pure-tone pairings were identical (e.g., $1000 \mathrm{~Hz}-1000 \mathrm{~Hz}$ ); the remainder differed by either a relatively small or relatively large amount, reflecting our manipulation of perceptual distance. It must be noted that the close versus far differences we operationally 
defined here are well beyond what audiologists have termed the "just noticeable difference" or minimal difference limen between tones. Studies in the older adult literature, for example, note that when pure tones range from $300 \mathrm{~Hz}$ to $2000 \mathrm{~Hz}$, any amplitude difference $>5 \mathrm{~dB}$ and frequency difference $>10 \mathrm{~Hz}$ tends to be perceptible by most older adults (Harris et al., 2007, 2008).

In the frequency block, tone pairs were either identical ( $n=16$ pairs), relatively close (i.e., $25 \mathrm{~Hz}$ ), or distant (i.e., $100 \mathrm{~Hz}$ ). Amplitude was matched using the process stated above, and duration was matched at $1000 \mathrm{~ms}$. For the trials that differed, half of the stimuli were ordered such that there was an increasing frequency difference (i.e., $500 \mathrm{~Hz}-525 \mathrm{~Hz}$ ); the remainder had a falling frequency difference $(525 \mathrm{~Hz}-500 \mathrm{~Hz})$.

In the amplitude block, tone pairs were either identical ( $n=16$ pairs), different by a small amount (i.e., $6 \mathrm{~dB} ; n=8$ pairs), or different by a large amount (i.e., $12 \mathrm{~dB} ; n=8$ pairs). We matched tone duration at $1000 \mathrm{~ms}$ and pitch at $1000 \mathrm{~Hz}$ during all trials. For the trials that differed, half of the stimuli were ordered such that there was a rising amplitude (i.e., $40 \mathrm{~dB}-$ $46 \mathrm{~dB})$; the remainder had a falling amplitude ( $46 \mathrm{~dB}-40 \mathrm{~dB})$.

In the duration block, tone pairs were either identical ( $n=16$ pairs), different by a small amount (i.e., $500 \mathrm{~ms} ; n=8$ pairs), or different by a large amount (i.e., $2000 \mathrm{~ms} ; n=8$ pairs). Tone frequency was matched at $1000 \mathrm{~Hz}$. We matched for intensity (perceived as volume) by batch processing the stimulus set to their root mean square amplitude using an acoustic waveform editor. This process was also employed to match intensity for the tones in the frequency block. For the trials that differed, half of the stimuli were ordered such that there was an increasing acoustic duration (i.e., $1000 \mathrm{~ms}-1500 \mathrm{~ms}$ ); the remainder had a decreasing acoustic duration (1500 ms-1000 ms).

\section{Data Analyses and Statistical Model}

The dependent measure in this forced-choice format was response accuracy. We converted errors and hit rates to $d^{\prime}$ scores in order to account for chance guessing (Wickens, 2002). $D^{\prime}$ scores can be defined as the standardized difference between the signal and noise. The participant's $d^{\prime}$ score was calculated as $Z$ hit rate (HR) minus $Z$ false alarm rate (FAR): $Z$ HR $-Z$ FAR. The HR was determined by dividing the number of true positives (TPs) by the sum of the number of TPs and false negatives (FNs). The FAR was determined by dividing the number of false positives (FPs) by the sum of the number of FPs and true negatives (TNs). TPs were defined as the participant correctly identifying the stimuli as different, FNs were defined as the participant incorrectly identifying the stimuli as the same when they were different, FPs were defined as the participant incorrectly identifying the stimuli as different when they were the same, and TNs were defined as the participant correctly identifying the stimuli as the same.

We then submitted each participant's $d^{\prime}$ scores to a $3 \times 3 \times 2$ mixed-model analysis of variance (ANOVA). The within-subjects factors in this ANOVA were acoustic property (i.e., frequency, amplitude, or duration) and perceptual distance (i.e., close or far). The between-subjects factor was diagnosis (PD or Control).

\section{Results}

An analysis of the interactions revealed a significant 3-way Acoustic Property $\times$ Distance $\times$ Diagnosis interaction, $F(2,100)=4.48, p<.05$. The 3-way interaction was the highest order interaction to be significant; therefore, three 2-way ANOVAs were completed to break down the 3-way interaction. 
There was a significant 2-way Distance $\times$ Diagnosis interaction when only data from the frequency condition was input, $F(1,25)=16.75, p<.05$, and when only data from the amplitude condition was input, $F(1,25)=5.24, p<.05$. The interaction was not significant for the 2-way ANOVA when only data from the duration condition was input, $F(1,25)=$. $05, p>.05$. To further break down the interaction, multiple $t$ tests were conducted. These $t$ tests revealed significant results when the frequency difference was perceptually close, $t(25)$ $=-3.80, p<.05$, and when the amplitude difference was perceptually close, $t(25)=-2.74, p$ $<.05$. All other tests were not significant. Therefore, participants with PD did significantly worse than age-matched controls in frequency and amplitude distinctions when the differences were perceptually close (see Figure 1). Table 1 represents a summary of the results and corresponding percentage accuracies.

\section{Discussion}

Participants with PD showed marked impairment relative to age-matched controls in discriminating low-level auditory stimuli that varied by acoustic features and perceptual similarity. More specifically, participants with PD demonstrated reduced ability to identify subtle frequency and amplitude distinctions while showing temporal discrimination ability comparable to controls. The selective preservation of timing cues was unexpected given the body of literature demonstrating a range of explicit time estimation and motor timing deficits in persons with PD (Harrington, Haaland, \& Hermanowicz, 1998; Jones, Malone, Dirnberger, Edwards, \& Jahanshahi, 2008; Pastor, Artieda, Jahanshahi, \& Obeso, 1992). These results support the hypothesis that persons with PD have decreased sensitivity to changes in at least some acoustic features of speech. One limiting factor for our findings, however, is the fact that our "close" duration measure may not have been as equally close as the close distinction for frequency and amplitude. This methodological limitation is largely imposed by the physical properties of sound in that scaling the difference between two orthogonal variables inherently requires estimation and a degree of subjectivity.

From a brain-behavior standpoint, PD is not a commonly regarded lesion model for auditory deficits. However, a rapidly evolving body of literature from both client-based case studies and functional neuroimaging implicates regions of the frontal lobes in auditory perception (Price et al., 1996; Tun, O'Kane, \& Wingfield, 2002). These findings raise several important questions and offer the potential for a number of unexplored avenues of research.

One unresolved issue with respect to PD is its challenge for the dual-stream model of speech perception. According to this influential model, the dorsal stream is composed of frontal lobe structures that are dedicated to sensorimotor integration of speech (Hickok, 2009; Hickok \& Poeppel, 2007). To a large extent, pure-tone presentation circumvents such auditory-visual crossmodal phenomena in that these stimuli are unlikely to immediately evoke complex articulatory motor plans. However, as discussed earlier, the frontal lobe has shown contributions with tone discrimination. Our findings suggest that these frontal lobe contributions might be susceptible to disease such as PD.

Another potential avenue of future research will involve elucidating the role of general cognitive and resource limitations (e.g., attentional vigilance, inhibitory control, resource allocation) in auditory perception in persons with PD. Our study revealed a general decreased sensitivity to change in auditory perception in persons with PD that was greater than the already decreased sensitivity seen in the healthy aging population. There is a vast body of literature in the healthy aging population that has demonstrated that peripheral deficits in sensory acuity interact in complex ways with more central cognitive processes (Anstey, Luszcz, \& Sanchez, 2001; Cattaneo, Bhatt, Merabet, Pece, \& Vecchi, 2008; Clay et al., 2009; Uhlmann, Larson, Rees, Koepsell, \& Duckert, 1989). There is also an extensive 
body of research demonstrating that PD produces a range of dysexecutive deficits that may compromise the cognitive contribution to sensory processing (Emre, 2004; Padovani, Costanzi, Gilberti, \& Borroni, 2006). Therefore, further investigation into the loci of auditory deficits in persons with PD will benefit from an expanded sample size, sensitive measures of audition, and quantitative correlations with neuropsychological measures of executive control. For example, the inclusion of a shorter ISI (closer to 200-300 ms) in this same task would reduce the auditory memory burden of the task and help elucidate whether this can improve sensitivity to change for acoustic properties of speech in persons with PD. Also, an auditory battery that was staged in successive complexity from pure tones through nonword minimal pairs (e.g., ba-ga) ascending to real words will allow researchers to better discern whether deficits in PD are "speech specific" or have an overarching processing basis. Deficits in pure-tone discrimination as we found here suggest both possibilities. However, the extent to which such lower level auditory deficits pervade higher level linguistic processes remains an open question.

Surprenant and Watson (2001) displayed evidence that increased acuity for acoustic features in adults did not correlate with increased performance on speech processing tasks. Although this raises important questions as to whether the tone discrimination difficulties seen in persons with PD have effects of speech processing, it is important to note that the previous study was conducted on healthy young adults; therefore, we can at the very least conclude that an intact tone discrimination system does not correlate well with speech processing but may not extend to the diseased system that is likely seen in persons with PD.

In conclusion, we found that persons with PD showed a decreased ability to notice small changes in frequency and amplitude of pure tones. Further testing, however, is needed to better dissociate the role of executive resources using more neuropsychological testing and also to extend this research into this phenomenon and its possible role in speech processing. With further research into these questions, we may be able to better understand this phenomenon for purposes of treatment but also for a better understanding of fronto-striatal portions of the brain in tone and speech processing.

\section{Acknowledgments}

This work was supported by U.S. Public Health Service Grants K23 DC010197 (JR) and NS53488, AG15116, NS44266, and AG17586 (MG).

\section{References}

Abbruzzese G, Berardelli A. Sensorimotor integration in movement disorders. Movement Disorders. 2003; 18(3):231-240. [PubMed: 12621626]

Anstey KJ, Luszcz MA, Sanchez L. A reevaluation of the common factor theory of shared variance among age, sensory function, and cognitive function in older adults. The Journals of Gerontology Series B: Psychological Sciences and Social Sciences. 2001; 56(1):P3-P11.

Beck AT, Steer RA, Carbin MG. Psychometric properties of the Beck Depression Inventory: Twentyfive years of evaluation. Clinical Psychology Review. 1988; 8(1):77-100.

Binder JR, Frost JA, Hammeke TA, Bellgowan PS, Springer JA, Kaufman JN, Possing ET. Human temporal lobe activation by speech and nonspeech sounds. Cerebral Cortex. 2000; 10(5):512-528. [PubMed: 10847601]

Binder JR, Frost JA, Hammeke TA, Rao SM, Cox RW. Function of the left planum temporale in auditory and linguistic processing. Brain. 1996; 119(4):1239-1247. [PubMed: 8813286]

Braak H, Ghebremedhin E, Rub U, Bratzke H, Del Tredici K. Stages in the development of Parkinson's disease-related pathology. Cell and Tissue Research. 2004; 318(1):121-134. [PubMed: 15338272] 
Burton MW, Small SL. Functional neuroanatomy of segmenting speech and nonspeech. Cortex. 2006; 42(4):644-651. [PubMed: 16881272]

Caspari, I. Wernicke's aphasia. In: LaPointe, LL., editor. Aphasia and related neurogenic language disorders. 3. Stuttgart, Germany: Thieme; 2005. p. 142-168.

Cattaneo Z, Bhatt E, Merabet LB, Pece A, Vecchi T. The influence of reduced visual acuity on agerelated decline in spatial working memory: An investigation. Aging, Neuropsychology, and Cognition. 2008; 15(6):687-702.

Clay OJ, Edwards JD, Ross LA, Okonkwo O, Wadley VG, Roth DL, Ball KK. Visual function and cognitive speed of processing mediate age-related decline in memory span and fluid intelligence. Journal of Aging and Health. 2009; 21(4):547-566. [PubMed: 19436063]

Damasio, H. Neural basis of language disorders. In: Chapey, R., editor. Language intervention strategies in aphasia and related neurogenic language disorders. 4. Baltimore, MD: Lippincott Williams \& Wilkins; 2001. p. 18-36.

Demirci M, Grill S, McShane L, Hallett M. A mismatch between kinesthetic and visual perception in Parkinson's disease. Annals of Neurology. 1997; 41(6):781-788. [PubMed: 9189039]

Emre M. Dementia in Parkinson's disease: Cause and treatment. Current Opinion in Neurology. 2004; 17(4):399-404. [PubMed: 15247534]

Folstein MF, Folstein SE, McHugh PR. "Mini-Mental State": A practical method for grading the cognitive state of patients for the clinician. Journal of Psychiatric Research. 1975; 12(3):189-198. [PubMed: 1202204]

Gawel MJ, Das P, Vincent S, Rose FC. Visual and auditory evoked responses in patients with Parkinson's disease. Journal of Neurology, Neurosurgery \& Psychiatry. 1981; 44(3):227-232.

Graber S, Hertrich I, Daum I, Spieker S, Ackermann H. Speech perception deficits in Parkinson's disease: Underestimation of time intervals compromises identification of durational phonetic contrasts. Brain and Language. 2002; 82(1):65-74. [PubMed: 12174816]

Harrington DL, Haaland KY, Hermanowicz N. Temporal processing in the basal ganglia. Neuropsychology. 1998; 12(1):3-12. [PubMed: 9460730]

Harris KC, Mills JH, Dubno JR. Electro-physiologic correlates of intensity discrimination in cortical evoked potentials of younger and older adults. Hearing Research. 2007; 228(1-2):58-68. [PubMed: 17344001]

Harris KC, Mills JH, He NJ, Dubno JR. Age-related differences in sensitivity to small changes in frequency assessed with cortical evoked potentials. Hearing Research. 2008; 243(1-2):47-56. [PubMed: 18597958]

Hickok G. The functional neuroanatomy of language. Physics of Life Reviews. 2009; 6(3):121-143. [PubMed: 20161054]

Hickok G, Poeppel D. The cortical organization of speech processing. Nature Reviews Neuroscience. 2007; 8(5):393-402.

Hoehn MM, Yahr MD. Parkinsonism: Onset, progression, and mortality. Neurology. 1967; 17(5):427442. [PubMed: 6067254]

Jones CRG, Malone TJL, Dirnberger G, Edwards M, Jahanshahi M. Basal ganglia, dopamine and temporal processing: Performance on three timing tasks on and off medication in Parkinson's disease. Brain and Cognition. 2008; 68(1):30-41. [PubMed: 18378374]

Kaplan, E.; Goodglass, H.; Weintraub, S. The Boston Naming Test. Philadelphia, PA: Lea and Febiger; 1983.

Kertesz A, Lau WK, Polk M. The structural determinants of recovery in Wernicke's aphasia. Brain and Language. 1993; 44(2):153-164. [PubMed: 8428309]

Klockgether T, Borutta M, Rapp H, Spieker S, Dichgans J. A defect of kinesthesia in Parkinson's disease. Movement Disorders. 1995; 10(4):460-465. [PubMed: 7565827]

Kotz SA, Schwartze M, Schmidt-Kassow M. Non-motor basal ganglia functions: A review and proposal for a model of sensory predictability in auditory language perception. Cortex. 2009; 45(8):982-990. [PubMed: 19361785]

Liberman AM, Cooper FS, Shankweiler DP, Studdert-Kennedy M. Perception of the speech code. Psychological Review. 1967; 74(6):431-461. [PubMed: 4170865] 
McGurk H, MacDonald J. Hearing lips and seeing voices. Nature. 1976; 264(5588):746-748. [PubMed: 1012311]

Muthane UB, Satishchandra P, Subhash MN. Visual and auditory evoked potentials in early onset Parkinson's disease and their relationship to cerebrospinal fluid monoamine metabolites. Movement Disorders. 1993; 8(3):344-348. [PubMed: 7688076]

Padovani A, Costanzi C, Gilberti N, Borroni B. Parkinson's disease and dementia. Neurological Sciences. 2006; 27(Suppl):S40-S43. [PubMed: 16708183]

Pastor MA, Artieda J, Jahanshahi M, Obeso JA. Time estimation and reproduction is abnormal in Parkinson's disease. Brain. 1992; 115(Pt 1):211-225. [PubMed: 1559155]

Pell MD. On the receptive prosodic loss in Parkinson's disease. Cortex. 1996; 32(4):693-704. [PubMed: 8954247]

Pell MD, Cheang HS, Leonard CL. The impact of Parkinson's disease on vocal-prosodic communication from the perspective of listeners. Brain and Language. 2006; 97(2):123-134. [PubMed: 16226803]

Pell MD, Leonard CL. Processing emotional tone from speech in Parkinson's disease: A role for the basal ganglia. Cognitive, Affective \& Behavioral Neuroscience. 2003; 3(4):275-288.

Pichora-Fuller MK, Souza PE. Effects of aging on auditory processing of speech. International Journal of Audiology. 2003; 42(6 Supp 2):11-16.

Price CJ, Wise RJ, Warburton EA, Moore CJ, Howard D, Patterson K, Friston KJ. Hearing and saying. The functional neuro-anatomy of auditory word processing. Brain. 1996; 119(Pt 3):919-931. [PubMed: 8673502]

Reilly J, Troiani V, Grossman M, Wingfield A. An introduction to hearing loss and screening procedures for behavioral research. Behavior Research Methods. 2007; 39(3):667-672. [PubMed: 17958180]

Schneider JS, Diamond SG, Markham CH. Deficits in orofacial sensorimotor function in Parkinson's disease. Annals of Neurology. 1986; 19(3):275-282. [PubMed: 3963772]

Smith JG, Harper DN, Gittings D, Abernethy D. The effect of Parkinson's disease on time estimation as a function of stimulus duration range and modality. Brain and Cognition. 2007; 64(2):130-143. [PubMed: 17343966]

Sprengelmeyer R, Young AW, Mahn K, Schroeder U, Woitalla D, Büttner T, Przuntek H. Facial expression recognition in people with medicated and unmedicated Parkinson's disease. Neuropsychologia. 2003; 41(8):1047-1057. [PubMed: 12667540]

Surprenant AM, Watson CS. Individual differences in the processing of speech and nonspeech sounds by normal-hearing listeners. The Journal of the Acoustical Society of America. 2001; 110(4): 2085-2095. [PubMed: 11681386]

Tun PA, O'Kane G, Wingfield A. Distraction by competing speech in young and older adult listeners. Psychology and Aging. 2002; 17(3):453-467. [PubMed: 12243387]

Uhlmann RF, Larson EB, Rees TS, Koepsell TD, Duckert LG. Relationship of hearing impairment to dementia and cognitive dysfunction in older adults. JAMA. 1989; 261(13):1916-1919. [PubMed: 2926927]

Wernicke, C. Der aphasische symptomemkomplex: Eine psychologische Studie auf anatomischer basis [The aphasia symptom-complex: A psychological study on an anatomical basis]. Vienna, Austria: Cohn und Weigert; 1874.

Wickens, TD. Elementary signal detection theory. New York, NY: Oxford University Press; 2002. 


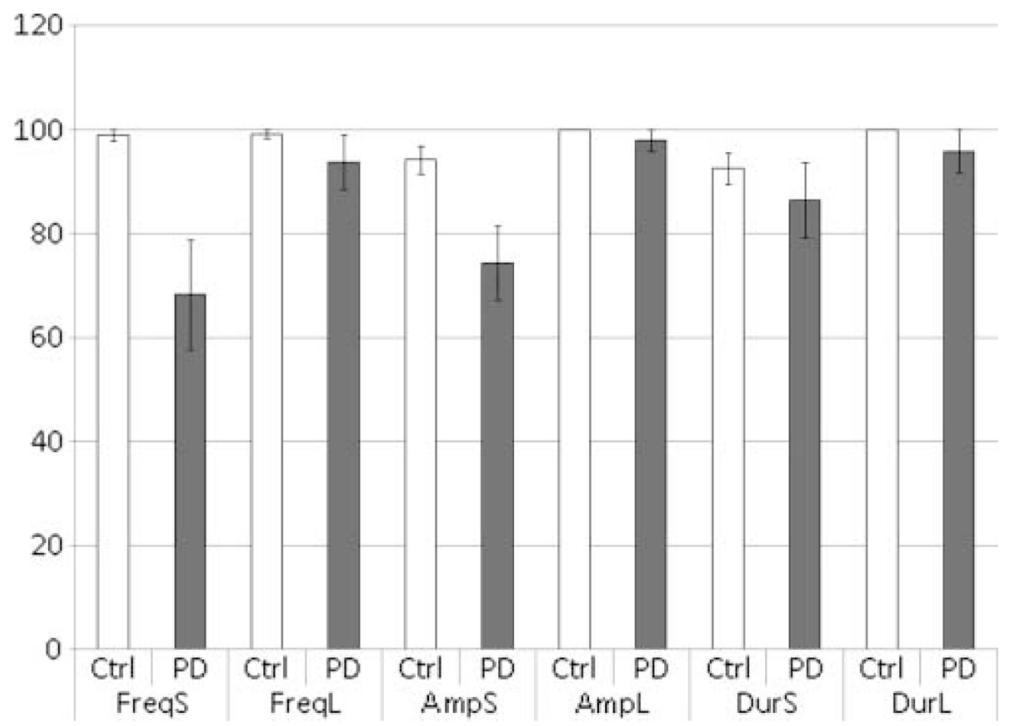

FIGURE 1. Percentage correct across the participants' diagnosis, distance, and acoustic property

Note . Ctrl = Control, $\mathrm{PD}=$ Parkinson's disease, FreqS = frequency with small perceptual distance, FreqL = frequency with large perceptual distance, $\mathrm{AmpS}=$ amplitude with small perceptual distance, AmpL = amplitude with large perceptual distance, DurS = duration with small perceptual distance, DurL $=$ duration with large perceptual distance. 


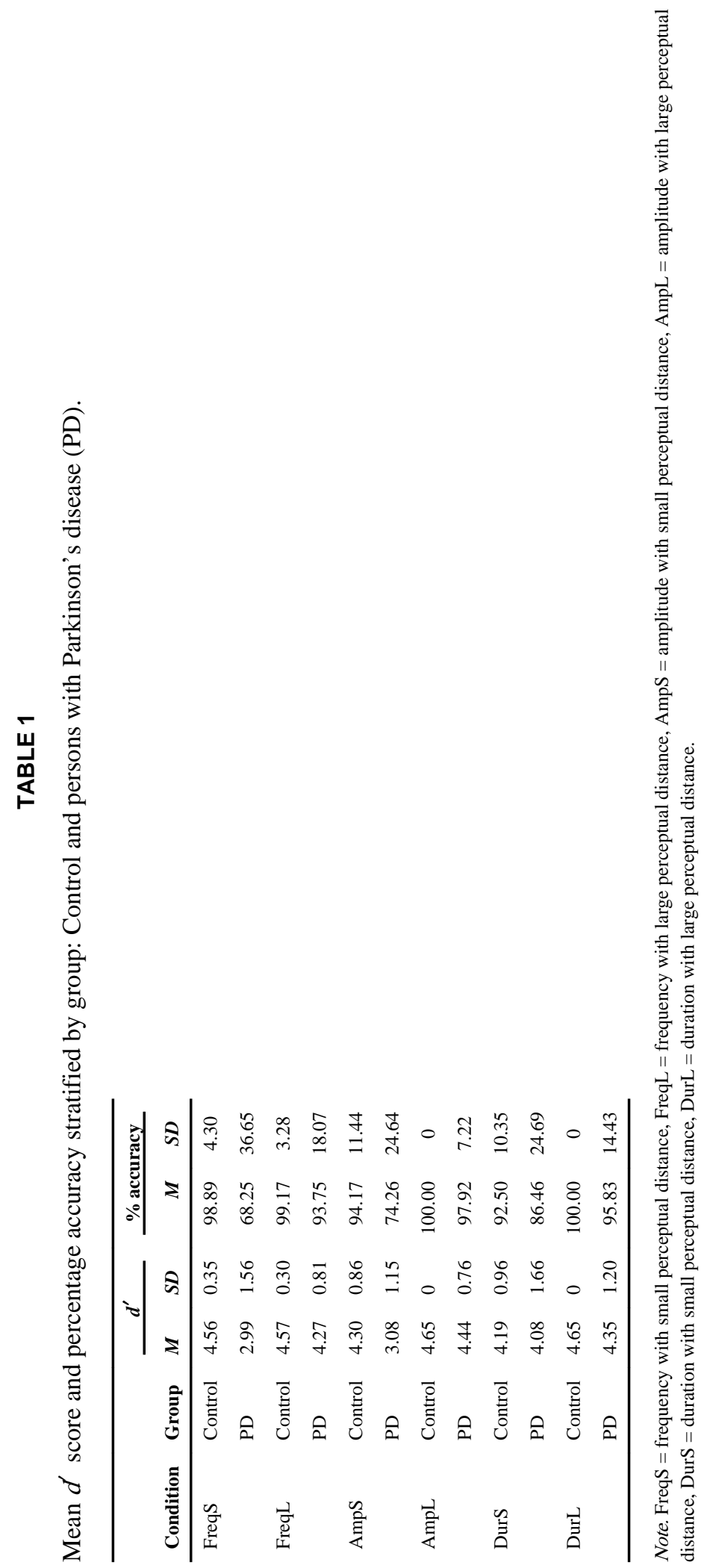

